\title{
Green Refrigerator Implementation using Peltier Cell and GSM
}

\author{
Sushanth K J' ${ }^{1}$, Asif Iqbal Mulla ${ }^{1}$, Suhail Mohammad², Mohammad Ijaz ${ }^{2}$, Usha T.N ${ }^{2}$, Muhammed Nibrasudeen ${ }^{2}$ \\ Assistant Professor, Electronics and Communication Engineering Department, BIT, Mangalore, Karnataka, India ${ }^{1}$ \\ Students of Final Year, Electronics and Communication, Bearys Institute of Technology, Mangaluru, Karnataka, India ${ }^{2}$
}

\begin{abstract}
The Peltier cell replaces the bulky compressors in a typical refrigerator. We designed and constructed a compressor-less thermoelectric refrigerator that uses Peltier cells for cooling. A temperature sensor is used to measure the temperature inside the fridge that gives an accuracy of $+/-0.05^{\circ} \mathrm{C}$. Our main aim is to promote green environment with our project work. That is using Peltier cells instead of refrigerants that are harmful to the environment. Our project work is compact. It has a GSM module to control or set the temperature inside the refrigerator and also a Wi-Fi module to monitor it. LCD is also used to monitor it near the system. We also experimented to find out that out project work was able to bring the temperature $27^{\circ} \mathrm{C}$ down to $17^{\circ} \mathrm{C}$ inside a room.
\end{abstract}

Keywords: Peltier cell, GSM and Wi-Fi module.

\section{INTRODUCTION}

Thermoelectric refrigerator sometimes called a thermoelectric cooler module or Peltier cooler is a semiconductor based electric component that functions as a small heat pump. By applying a low voltage direct current (DC) power source to a thermoelectric cooler module, heat will be moved through the module from one side to the other. One module face, therefore, will be cooled while the opposite face simultaneously is heated. The application of Direct Current (DC) power to the thermoelectric cooler modules causes electrons to move through the semi-conductor material. At the cold end of the semi-conductor material, heat is absorbed by the electron movement, moved through the material, and expelled at the hot end. Since the hot end of the material is physically attached to a heat sink, the heat is passed from the material to the heat sink and then in turn, transferred to the environment.

\section{METHODOLOGY AND PROPOSED SYSTEM}

The Proposed system is implementation of thermoelectric refrigerator (Green Refrigerator). Peltier Cell is used for cooling and DS18B20 is used for sensing temperature. Wi-Fi and GSM is used to monitor and control the temperature within the refrigerator. Relays is used to switch the cooling fans ON or OFF. LCD is used to monitor the temperature inside the refrigerator near it. Programming software that is used to program the Arduino Uno is ARDUINO IDE. Application that is used to monitor the system in smartphone is TCP/UDP Terminal. The system is also monitored using 16X2 LCD and also it can be monitored using serial monitor directly using the PC. GSM is also used to set the threshold temperature.

\section{DESIGN AND IMPLEMENTATION}

Peltier thermoelectric cells (TEC) are solid-state devices that works on the principle of thermoelectric effect.
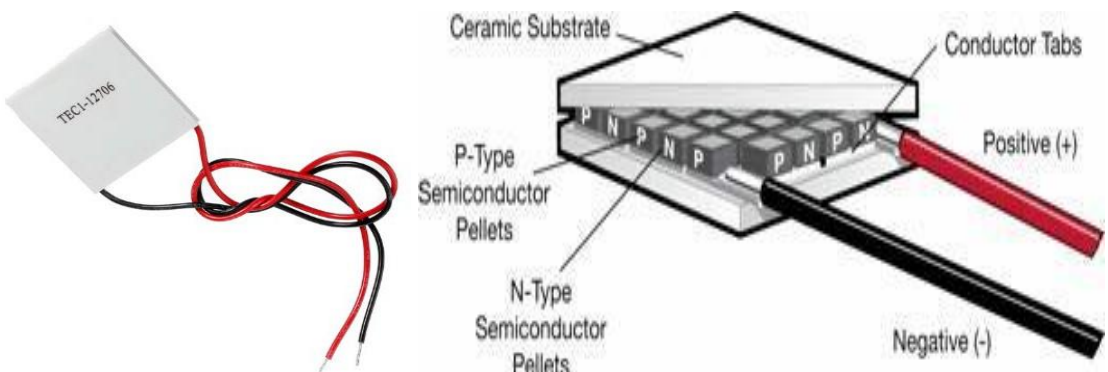

Fig.1 Peltier cell TEC1-12706 


\section{International Journal of Innovative Research in Electrical, Electronics, Instrumentation and Control Engineering \\ ISO 3297:2007 Certified}

Vol. 5, Issue 6, June 2017

The thermoelectric effect is the direct conversion of temperature differences to electric voltage and vice versa. A thermoelectric device creates voltage when there is a different temperature on each side. Conversely, when a voltage is applied to it, it creates a temperature difference. At the atomic scale, an applied temperature gradient causes charge carriers in the material to diffuse from the hot side to the cold side as shown in fig1.

The thermoelectric effect is much larger at metal-semiconductor junction than in a junction between two metals. In the world of thermoelectric technology, semiconductor (usually BismuthTelluride) are the material of choice for producing the Peltier effect.

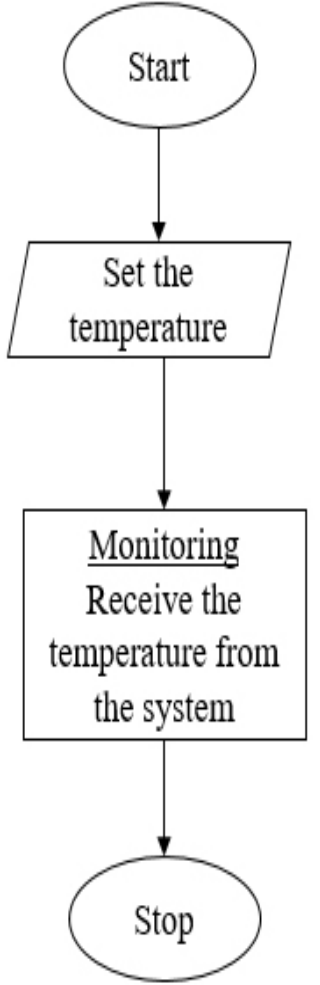

Fig. 2: Used end

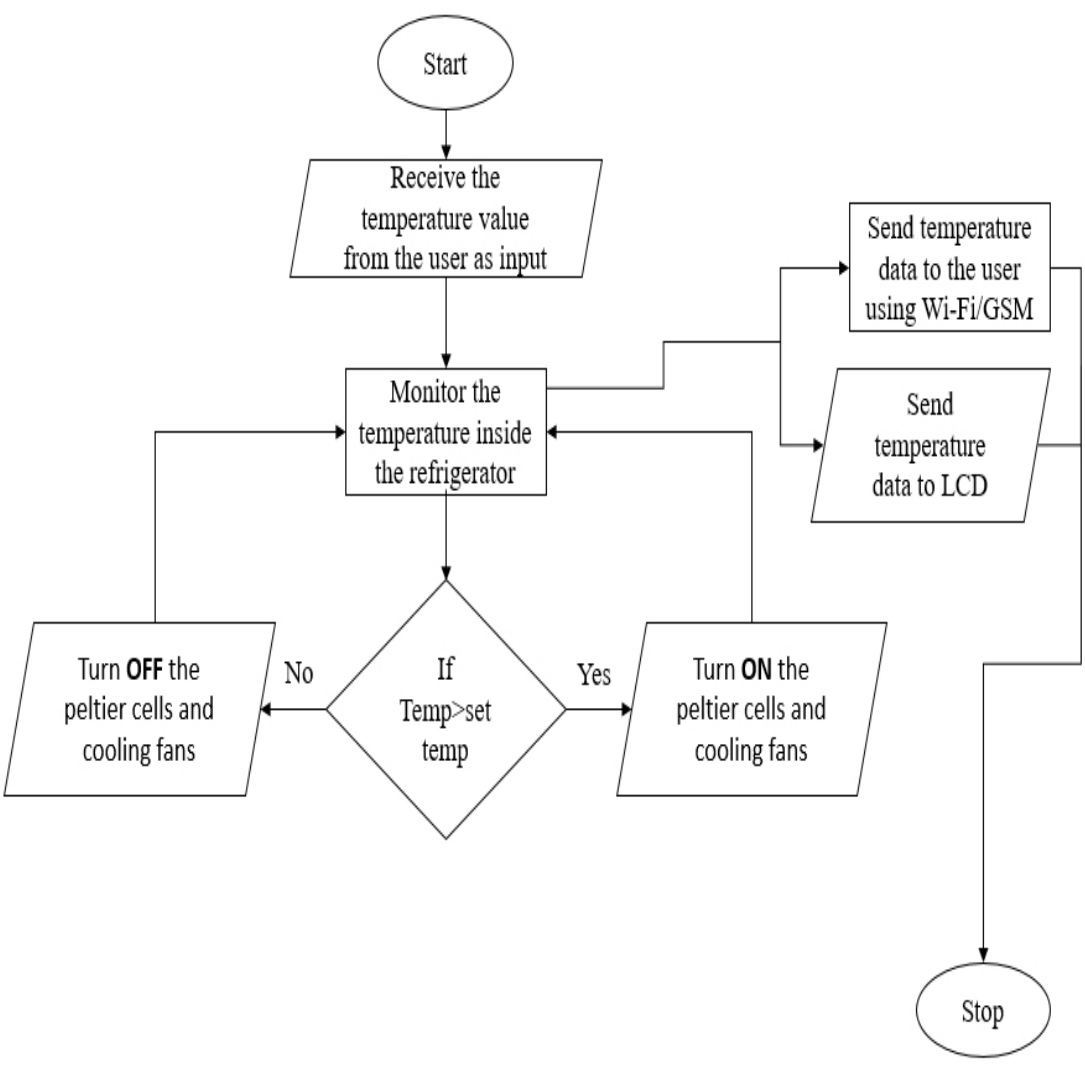

Fig. 3 : System end

The set temperature will be received by the system and it will be considered as threshold. The system will continuously monitor the temperature using the DS18B20 temperature sensor. This data is sent to the user as well as the LCD.

If the internal temperature of the refrigerator is more than the threshold then, the relay will switch the Peltier cell to cool down the system to the set temperature as shown in fig 2 and 3. On achieving the temperature, the Peltier will be turned off but the cooling fans will still be running in order to dissipate the heat outside because the Peltier will still be hot on one side.

On switching off the Peltier cell, the system temperature will start increasing gradually. But the cells will not be turned on immediately. It will be turned on again once it reaches Threshold $+1^{\mathrm{O}} \mathrm{C}$ temperature. Similar to the method that is observed in Air conditioners. Or else the system will be continuously switching on and off the Peltier, which is not a proper methodology.

\section{EXPERIMENTAL OBSERVATIONS AND RESULTS}

\section{EXPERIMENTAL SETUP}

The overall top and side view of the model is shown in this section as well as the system initialization as shown in fig 4 and 5. 


\section{International Journal of Innovative Research in Electrical, Electronics, Instrumentation and Control Engineering \\ ISO 3297:2007 Certified}

Vol. 5, Issue 6, June 2017

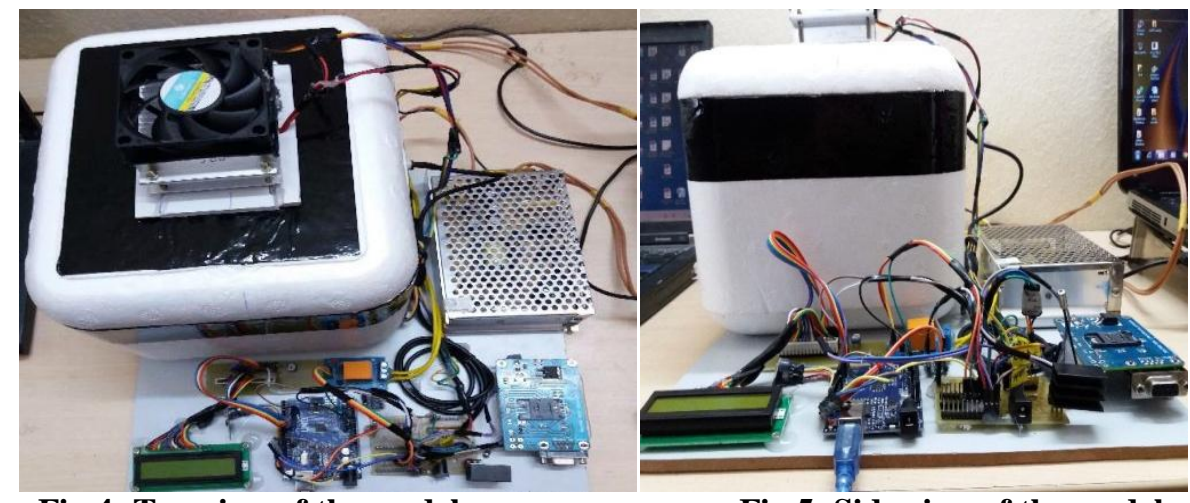

Fig 4: Top view of the model

Fig 5: Side view of the model

\section{OBSERVATIONS AND RESULTS}

The below figure shows the initialization of LCD where the information of the Wi-Fi module and GSM module is being done. On initialization, the two cooling fans will start to operate.

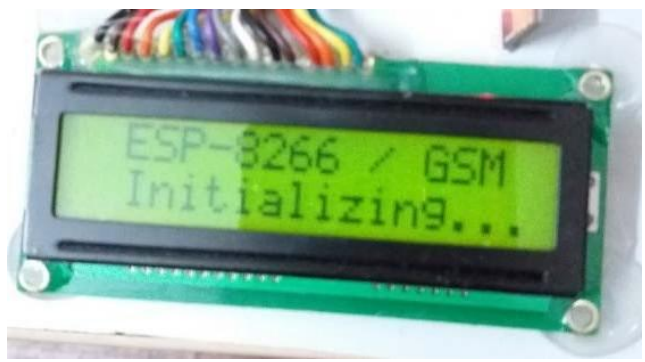

(a)

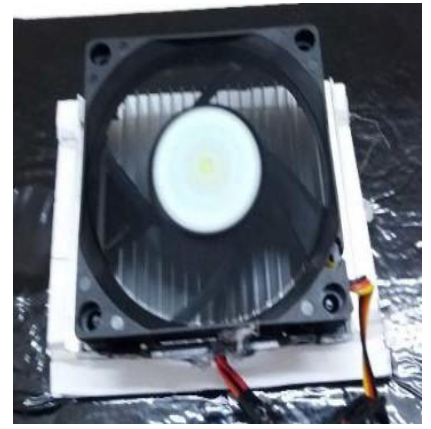

(b)

Fig.6 System Initialization

(b) Fig.7 cooling fans running

Next step Fig. 6 and Fig 7 is to initialize the system in the smartphone application where the temperature is continuously monitored. The smartphone must be connected to the module via Wi-Fi. The Wi-Fi details is given below. On entering this, the Wi-Fi application will be initialized and the temperature readings will be received for every 10 seconds. The following figure shows the initial temperature and final temperature achieved by our project work.
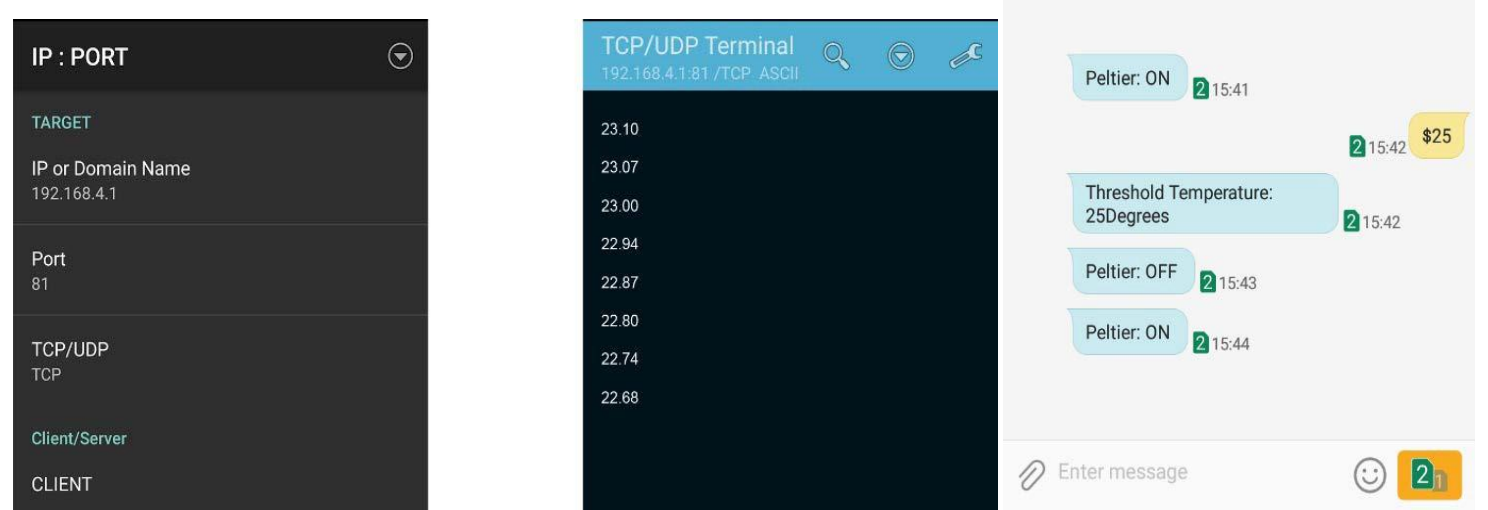

Fig.8: Wi-Fi application Initialization and Temperature data monitoring

In the case where the threshold is required, an SMS needs to be sent to the registered number. We will get an acknowledgement regarding ON/OFF of the Peltier. The threshold must be sent and the system will switch on the cells till the temperature is achieved. On reaching the threshold, the Peltier cell will be turned OFF and an acknowledgement will be sent back.

The first message is received by the user saying that the system is ON. Then a threshold of $25^{\circ} \mathrm{C}$ is set and an acknowledgement received. On achieving the $25^{\circ} \mathrm{C}$ mark, the Peltier is turned OFF an acknowledgement is sent again. 


\section{International Journal of Innovative Research in Electrical, Electronics, Instrumentation and Control Engineering \\ ISO 3297:2007 Certified \\ Vol. 5, Issue 6, June 2017}

\section{CONCLUSION AND FUTURE PERSPECTIVE}

This project work identifies the importance of green technology. How the technology can be used in a step towards the green revolution. In this project work, we have used Peltier cell that produces heat as well as cold and the cold is harnessed to use as a refrigerator. The remotely controlling of the device helps it in various fields of science as well as for the commercial purpose. Light weight and elimination of moving parts in this system make it more reliable and the maintenance also become very easy. The future lies bright for the thermoelectric devices. Since earth is at the urge of global warming, people tend to move towards the greener side. Researches are going on to make better and more efficient Peltier cells and also for its large-scale implementation. It is predicted that future refrigerators will run using Peltier cells and Cool chips.

\section{REFERENCES}

[1] Astrain D and Vian J G (2005), "Computational Model for Refrigerators Based on Peltier Effect Application”, Applied Thermal Engineering,

[2] Christian J L and Jadar R Barbosa Jr (2011), "Thermodynamic Comparison of Peltier, Stirling, and Vapor Compression Portable Coolers", Applied Energy

[3]. Roy J Dossat (2002), Principles of Refrigeration, Vol. 2

\section{BIOGRAPHIES}
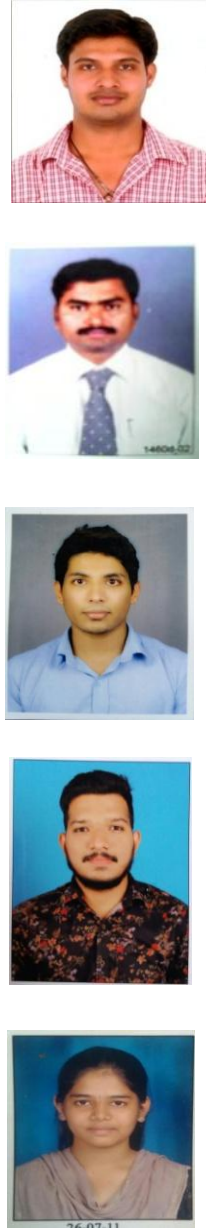

Sushanth K J received his BE from Coorg Institute of Technology, Coorg, India and M.Tech Degree from Sri Jayachamarajendra college of Engineering (SJCE), Mysore, under VTU, Karnataka, in the 2010 and 2012, respectively. At present he is working as Assistant Professor in Bearys Institute of Technology, Mangalore, India, in the department of Electronics and Communication Engineering. His research areas include Wireless Sensors networks, Embedded systems, and VLSI design.

Asif Iqbal. M. Mulla Received his BE from Anjuman Institute of Technology Bhatkal, Karnataka, India and M.Tech Degree from B.V. Bhoomaraddi college of Engineering (BVBCET), Hubli, under VTU, India, in the 2007 and 2013, respectively. He has Six years teaching and Three Years Industrial experience. At present he is working as assistant professor in Bearys Institute of Technology, Mangalore, India, in the department of Electronics and Communication Engineering. His research areas include Embedded systems and VLSI design.

Suhail Mohammad, Currently Pursuing BE, Final year in Electronics and Communication, at Bearys Institute of Technology, mangaluru, karnataka. His research interested in Embedded systems, Image Processing. Wireless networks.

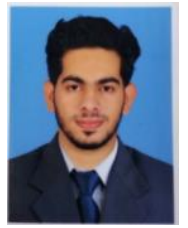

Muhammed Nibrasudeen, Currently Pursuing BE, Final year in Electronics and Communication, at Bearys Institute of Technology, mangaluru, karnataka. His research interested in Embedded systems, Image Processing. Wireless networks. 